\title{
Design of a Database-Driven GMV Controller Using the Nelder-Mead Method*
}

\author{
Liying Shi \\ Graduate School of Engineering, Hiroshima University, Japan \\ 1-4-1 Kagamiyama HigashiHiroshima ,739-8527, Japan \\ E-mail:m192383@hiroshima-u.ac.jp \\ Zhe Guan \\ KOBELCO Construction Machinery Dream-Driven Co-Creation Research Center, Hiroshima University, Japan \\ 1-4-1 Kagamiyama HigashiHiroshima ,739-8527, Japan \\ E-mail: guanzhe@hiroshima-u.ac.jp \\ Toru Yamamoto \\ Graduate School of Advanced Science and Engineering, Hiroshima, Japan \\ 1-4-1 Kagamiyama HigashiHiroshima ,739-8527, Japan \\ E-mail:yama@hiroshima-u.ac.jp
}

\begin{abstract}
This paper presents a design scheme that can obtain the optimized generalized minimum variance (GMV) control parameters by applying the Nelder-Mead (NM) method based on proportional-integral-derivative (PID) controller. The NM method is used to find the most suitable parameter $\lambda$. The application of NM method can optimize the calculation of $\lambda$. Furthermore, the estimation of closed-loop response method is introduced in database-driven approach. The effectiveness is verified by using a simulation example.
\end{abstract}

Keywords: PID controller, GMV, Nelder-Mead method, Database-driven approach

\section{Introduction}

A Proportional-Integral-Derivative (PID) controller ${ }^{1,2}$ is a three-term controller that has a long history in the automatic control field, and it is certainly the most widely used control scheme today owing to its intuitiveness and its relative simplicity. Over the last half-century, a great deal of academic and industrial effort has focused on improving PID control. In previous controller, using the minimum variance $(\mathrm{MV})^{3,4}$ as the benchmark can claim to be successful. The basic idea behind the MV index is to only consider the controller error variance. However, few of the available techniques in use take the control effort or the manipulative variable activity into account.
Then, the GMV ${ }^{5}$ is proposed that takes into account controller error variance as well as the manipulating variable variance. Furthermore, an adjustable parameter $\lambda$ (a weighting factor penalty on the manipulating variable) is included in the GMV. In the previous GMV controller, the PID parameters are calculated by simply changing $\lambda$ manually. Therefore, it is hard to get desirable control performance. In order to improve the control performance, the Nelder-Mead method ${ }^{6}$ is introduced. It can optimize the calculation of the most suitable parameter $\lambda$.

In this paper, a new GMV control scheme is proposed in which the controller parameters can be calculated without any model of the process by applying the Nelder- 
Mead method ${ }^{6}$ and method of the estimation of closedloop response ${ }^{7}$

The rest of this paper is organized as follows: the problem is formulated in section 2; the proposed scheme is the main topic in section 3 and section 4; the section 5 provides the simulation and analysis, and the section 6 concludes the paper.

\section{Overview of the Data-Driven GMV controller}

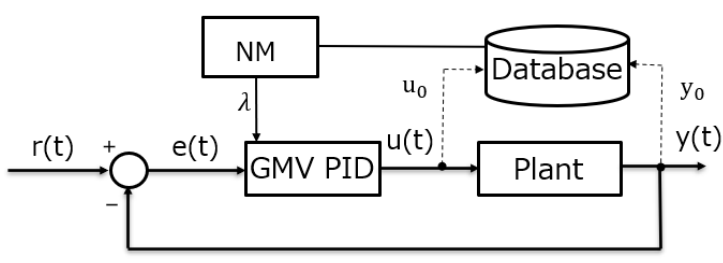

Fig. 1. The Block diagram of database-driven GMV controller.

In Fig. 1 , an adjustable parameter $\lambda$ is included in the GMV, and PID parameters are calculated by simply changing $\lambda$. Using the method of estimation of closedloop response to predict new output, then the application of NM method can optimize the calculation of the most suitable $\lambda$ according to the predict output and get optimal PID parameters without any model of the process.

\section{THE GENERALIZED MINIMUM VARIANCE CONTROL OF PARAMETERS}

\subsection{System description}

Consider the following process model and design of PID controller:

$$
A\left(z^{-1}\right) y(t)=z^{-1} B\left(z^{-1}\right) u(t)+\frac{\xi(\mathrm{t})}{\Delta}
$$

where $A\left(z^{-1}\right)$ and $B\left(z^{-1}\right)$ are given by the following polynomials:

$$
\begin{gathered}
A\left(z^{-1}\right)=1+a_{1} z^{-1}+a_{2} z^{-2} \\
\mathrm{~B}\left(\mathrm{z}^{-1}\right)=\mathrm{b}_{0}+\cdots+\mathrm{b}_{\mathrm{m}} \mathrm{z}^{-\mathrm{m}}
\end{gathered}
$$

where $u(t)$ and $y(t)$ are respectively the input and the corresponding output; $\xi(\mathrm{t})$ is the Gaussian white noise which has zero mean; $z^{-1}$, the backshift operator which implies $z^{-1} y(t)=y(t-1) ; \Delta$, the differencing operator $\left(\Delta=1-z^{-1}\right)$; and $m$, the order of $B\left(z^{-1}\right)$.

In this paper, the PID controller is introduced as $\frac{C\left(z^{-1}\right)}{\Delta}$ in the following equation:

$$
u(t)=\frac{C\left(z^{-1}\right)}{\Delta}\{r(t)-y(t)\}=\frac{C\left(z^{-1}\right)}{\Delta} e(t)
$$

where $r(t)$ is the reference signal; $e(t)$, the control error.

\subsection{Benchmark of the Generalized Minimum Variance Controller}

The GMV control law for the system (1) can be derived by minimizing the following cost function:

$$
J=E\left[\phi^{2}(t+k+1)\right] .
$$

Here, $\phi(t+k+1)$ is the generalized output given by following equation: $\phi(t+k+1)=P\left(z^{-1}\right) y(t+k+1)+\lambda \Delta u(t)-$ $P(1) \omega(t)$

where $\omega(\mathrm{t})$ denotes the step reference signal, $k$ is the minimum time-lag which is estimated by an operator $(k$ is set as 0 when the time-lag is unknown). In addition, $\lambda$ is the weight coefficient for the variance of the control input and it is set by a user arbitrarily. Next, the following Diophantine equation is introduced.

$$
\begin{gathered}
P\left(z^{-1}\right)=\Delta E\left(z^{-1}\right) A\left(z^{-1}\right)+z^{-(1+k)} F\left(z^{-1}\right) \\
E\left(z^{-1}\right)=1+e_{1} z^{-1}+\ldots+e_{k} z^{-k} \\
F\left(z^{-1}\right)=f_{0}+f_{1} z^{-1}+f_{2} z^{-2}
\end{gathered}
$$

Moreover, $P\left(z^{-1}\right)$ is the design polynomial and it is designed based on the following equation. where, the order of $E\left(z^{-1}\right)$ and $F\left(z^{-1}\right)$ are set to decide these coefficients uniquely from $\Delta A\left(z^{-1}\right)$ and $P\left(z^{-1}\right)$.

$$
\begin{gathered}
P\left(z^{-1}\right)=1+p_{1} z^{-1}+p_{2} z^{-2} \\
p_{1}=-2 \exp \left(-\frac{\rho}{2 \mu}\right) \cos \frac{\sqrt{4 \mu-1}}{2 \mu} \rho \\
p_{2}=\exp \left(-\frac{\rho}{\mu}\right) \\
\rho:=T_{s} / \sigma \\
\mu:=0,25(1-\delta)+0.51 \delta
\end{gathered}
$$

$\sigma$ and $\delta$ are parameters which express the rise time and the damping property which are set by an operator respectively. Moreover, ( $\sigma$ denotes the rise time that the system output attains about $60 \%$ of the value of the step reference signal. From (1), (4) and (5), the predictive value of the generalized output after $k+1$ at $t$ can be obtained as following equation:

$\phi(t+k+1)=F\left(z^{-1}\right) y(t)+\left\{E\left(z^{-1}\right) B\left(z^{-1}\right)+\right.$ $\lambda\} \Delta u(t)-P(1) \omega(t)+E\left(z^{-1}\right) \xi(t+k+1)$.

The control law is described as the following equation:

$$
\Delta u(t)=\frac{P(1)}{G\left(z^{-1}\right)+\lambda} \omega(t)-\frac{F\left(z^{-1}\right)}{G\left(z^{-1}\right)+\lambda} y(t) .
$$

By replacing the polynomial $G\left(z^{-1}\right)$ in (15) by the steady-state term $G(1)$, the following equation can be obtained:

$$
\Delta u(t)=\frac{P(1)}{G(1)+\lambda} \omega(t)-\frac{F\left(z^{-1}\right)}{G(1)+\lambda} y(t) .
$$


Therefore, PID parameters can be calculated as follows:

$$
\begin{aligned}
& K_{P}=\frac{f_{1}+2 f_{2}}{G(1)+\lambda} \\
& K_{I}=\frac{f_{1}+f_{2}+f_{0}}{G(1)+\lambda} \\
& K_{D}=\frac{f_{2}}{G(1)+\lambda}
\end{aligned}
$$

In this method, the control performance is strongly depended on $\lambda$. Then the user-specified parameters $\lambda$ is calculated by using the NM method. The predicted output adopts the estimation of closed-loop response method without any model. The detail of this approach will not be given because of pages limitation. Please refer to the Ref. 7.

\section{Evolutionary Computation Based on The Nelder-Mead method}

The Nelder-Mead method ${ }^{6}$ is briefly explained is to calculate the user-specified parameter $\lambda$. In this paper, the objective function is determined by

$$
H\left(S_{i}\right)=\sum_{t=1}^{M}\left\{y_{m}(t)-y_{d}(t)\right\}^{2}
$$

where $y_{m}(t)$ denotes the reference model output and $\mathrm{M}$ denotes the evaluation horizon. $y_{d}(t)$ is the predicted output corresponding to $S_{i}$. The objective function can be determined. The top with the largest target function value $H\left(S_{i}\right)$, the second large top and the smallest top are respectively assumed to be $S_{H}, S_{M}$ and $S_{L}$. Furthermore, the center between $S_{M}$ and $S_{L}$ is determined as $S_{G}$. Four operations are briefly explained as follows:

\section{- Reflection}

$S_{R}$ is determined as $S_{R}=(1+\beta) S_{G}-\beta S_{H}$, where $\beta$ is set as $\beta>0$, and it corresponds to the ratio of distances $S_{R} S_{G}$ and $S_{H} S_{G}$.

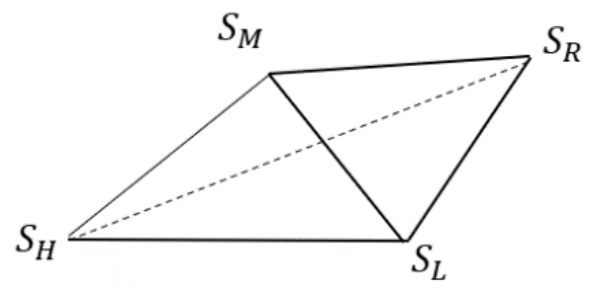

Fig. 2. Reflection of the Nelder-Mead method

- Expansion

$S_{E}$ is determined as $S_{E}=(1-\gamma) S_{G}+\gamma S_{H}$, where $\gamma$ is set as $\gamma>1$, and it corresponds to the ratio of distances $S_{E} S_{G}$ and $S_{R} S_{G}$.

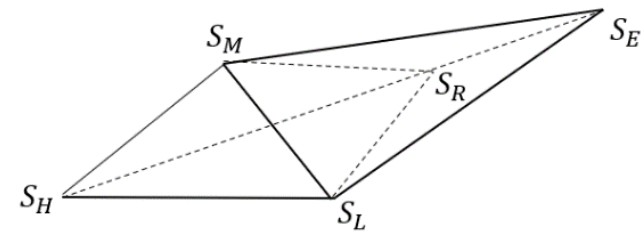

Fig. 3. Expansion of the Nelder-Mead method

- Contraction

$S_{C}$ is determined as $S_{C}=(1-\eta) S_{G}+\eta S_{H}$, where $\eta$ is set as $1>\eta>0$, and it corresponds to the ratio of distances $S_{C} S_{G}$ and $S_{H} S_{G}$.

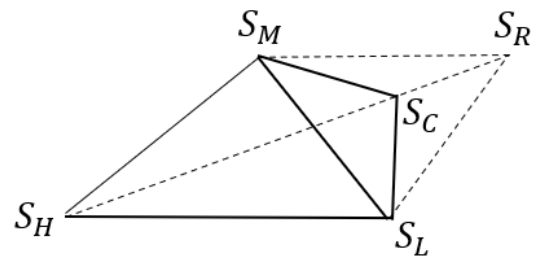

Fig. 4. the contraction of the Nelder-Mead method - Reduction

$S_{H}$ and $S_{M}$ are moved in the direction of $S_{L}$.

The proposed control system can be designed by calculate the most suitable $\lambda$ by the step based on the mentioned procedure.

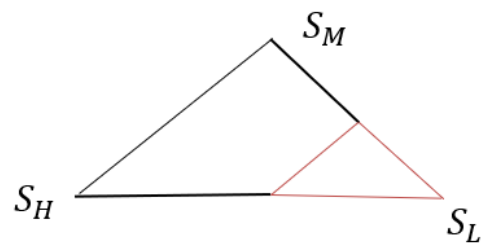

Fig. 5. Reduction of the Nelder-Mead method The proposed control system can be designed by calculating the most suitable $\lambda$ by the step based on the mentioned procedure.

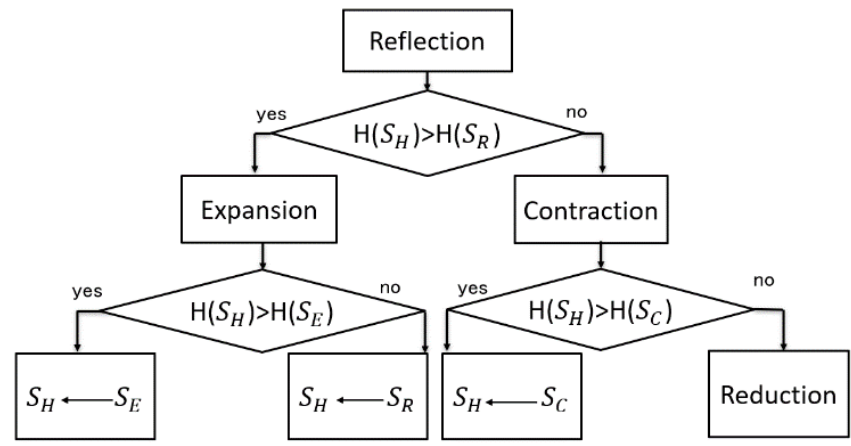

Fig.6. Procedure of the Nelder-Mead method 
If $H\left(S_{H}\right)>H\left(S_{R}\right)$, accept expansion, and then if $H\left(S_{H}\right)>H\left(S_{E}\right), S_{E}$ replace $S_{H}$. If $H\left(S_{H}\right)<H\left(S_{E}\right), S_{R}$ replace $S_{H}$. If $H\left(S_{H}\right)<H\left(S_{R}\right)$, accept contraction, and then if $H\left(S_{H}\right)>H\left(S_{C}\right), S_{C}$ replace $S_{H}$. If not, accept reduction.

\section{Simulation Example}

The effectiveness of the newly proposed design scheme is evaluated on a numerical simulation example.

$y(t)=-1.575 y(t-1)-0.654 y(t-2)+$

$0.023 u(t-1)-0.019 u(t-2)+\frac{\xi(\mathrm{t})}{\Delta}$

Firstly, Fig. 7 shows the control result by using manually adjusted $\lambda$.

when $\lambda=0.09$
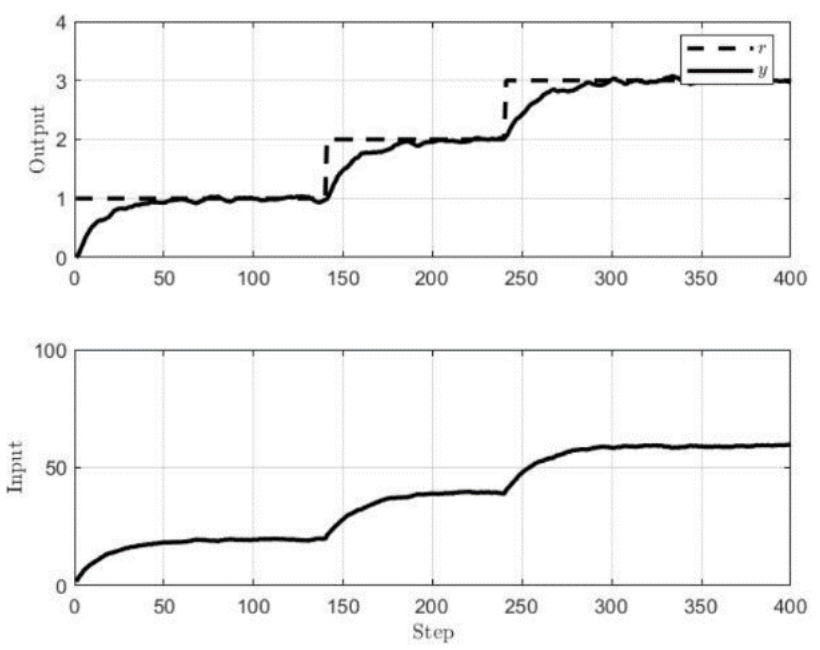

Fig. 7. Control result by using manually adjusted $\lambda$.

From the result, using manually adjusted $\lambda$ can not get desirable control performance. Then Fig.8 shows the control result by using the newly proposed method, where the design parameters of Nelder-Mead method are set as shown in Table 1.

Table 1. Parameters used in Nelder-Mead method.

\begin{tabular}{|c|c|c|c|c|}
\hline$S_{\max }$ & $S_{\min }$ & $\beta$ & $\gamma$ & $\eta$ \\
\hline$(1.0,1.0)$ & $(0.0,0.0)$ & 0.1 & 1.1 & 0.1 \\
\hline
\end{tabular}

Using the NM method, $\lambda=0.05$ is the most suitable parameter. Comparing with the two kinds of control results, it is clear that the newly proposed control scheme works well.
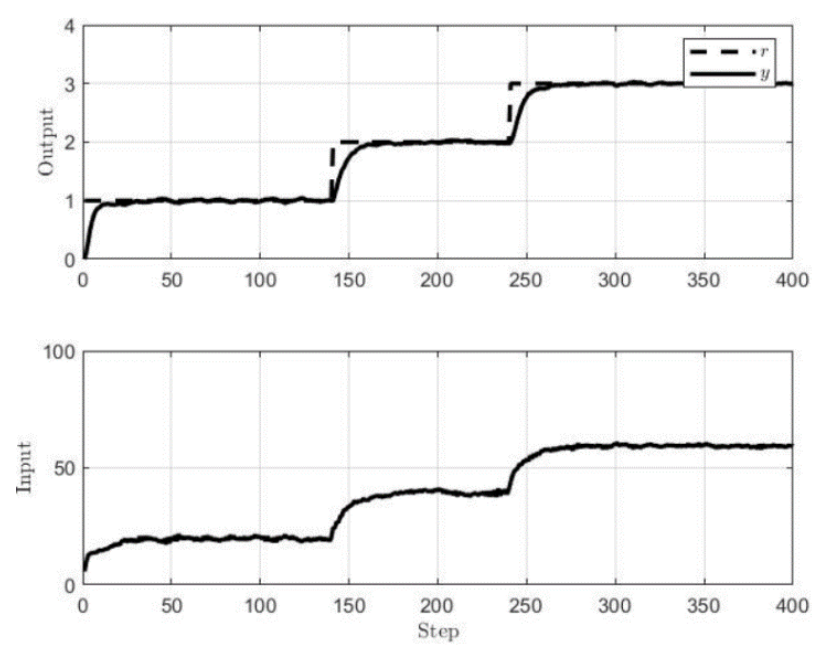

Fig. 8.the control result by using calculated $\lambda$.

\section{Conclusions}

In this paper, a design scheme of database-driven GMV controller has been proposed, which can obtain GMV control parameters by applying the NM method based on PID controller. The features of the newly proposed control scheme are summarized as follows:

- PID parameters are adjusted based on the GMV control system.

- The user-specified parameter included in GMV control system, is calculated by NM method for liner system.

- The estimation of closed-loop response is introduced in database-driven approach.

The effectiveness of the proposed scheme has been evaluated on the simulation example.

\section{References}

1. Michael A. J, Mohamman H. M. PID Control. SpringerVerlag, 2005.

2. Antonio V. Practical PID control. Springer-Verlag, 2006.

3. Harris, T.J. Assessment of closed loop performance. Can. J. Chem. Eng. 1989, pp.856-861.

4. S. Wakitani, S. Hanata, T. Yamamoto. Design and Application of a Direct GMV-Based PID Controller. Proceedings of the 2012 International Conference on Advanced Mechatronic Systems, 2012.

5. Astrom K. J; Hagglund T. The future of PID control. Control Engineering Practice 2001, pp.1163-1175.

6. Nelder J. A, Mead R. A Simplex Method for Function Minimization. Computer J. 1965, pp.100-313.

7. T. Sakatoku, K. Yubai, D. Yashiro, S. Komada. Estimation of Closed-Loop Response and Controller Retuning. IEEJapan, 2020. 